\title{
Modeling of Loss-Induced Superluminal and Negative Group Velocity in Two-Port Ring-Resonator Circuits
}

\author{
H. P. Uranus and H. J. W. M. Hoekstra
}

\begin{abstract}
The group velocity $v_{g}$ of light in two-port ringresonator (TPRR) circuits with loss is theoretically studied. We point out four possible operation regimes, i.e., "slow" light with positive $v_{g}$, "slow" light with negative $v_{g}$, "fast" light with negative $v_{g}$, and "fast" light with positive $v_{g}$, where "slow" means $\left|v_{g}\right|<c$ and "fast" means $\left|v_{g}\right|>c$, with $c$ denoting the velocity of light in vacuum. The temporal behavior of pulses passing through the TPRR for the four operation regimes and the potential of "slow" light TPRR either with positive or negative $v_{g}$ for sensing applications are also discussed.
\end{abstract}

Index Terms-Fast light, negative group velocity, ring resonator, sensor, slow light, superluminal group velocity.

\section{INTRODUCTION}

$\mathbf{R}$ ECENTLY, the ability to control the group velocity $v_{g}$ of light in dedicated media or structures has attracted the attention of many researchers [1]-[14]. "Slow" light, which is a phenomenon whereby light travels with a low $v_{g}$ (much lower than the light velocity in vacuum $c$ ), will enhance light-matter interaction and, hence, is prospective for applications such as sensing, nonlinear optics, light generation, and amplification, as well as applications such as optical delay lines and memory, which rely on the fact that light pulses are heavily delayed [1], [2]. In addition, light can also be controlled to travel with large $v_{g}$ (i.e., superluminal $v_{g}$, where $v_{g}$ is larger than $c$ ) or even with negative $v_{g}$ [1], [3]-[6], [8], [9]. In fact, superluminal and negative $v_{g}$ phenomena are not new subjects, as they have been theoretically studied since a few decades ago [12], [13] and have been observed experimentally, e.g., in photonic crystals [8], atomic gases [3], [4], active optical fibers [6], and lefthanded materials [14]. The consistency of such phenomena with causality has also been verified experimentally [5]. Such "slow" light or "fast" light, positive or negative $v_{g}$ phenomena can be attained through engineering the material dispersion of the medium [1], [3]-[7] or the structural dispersion of the waveguiding structures [2], [8]-[11].

Ring-resonator circuits, which are very dispersive around its resonant frequency, can exhibit "slow" light phenomenon [2],

Manuscript received January 13, 2007; revised May 8, 2007. This work was supported by the STW Technology Foundation under Project TOE 6596.

The authors are with the Integrated Optical MicroSystems Group, MESA+ Institute for Nanotechnology, University of Twente, 7500 AE Enschede, The Netherlands (e-mail: h.p.uranus@ewi.utwente.nl; h.j.w.m.hoekstra@ewi. utwente.nl).

Color versions of one or more of the figures in this paper are available online at http://ieeexplore.iee.org.

Digital Object Identifier 10.1109/JLT.2007.901524

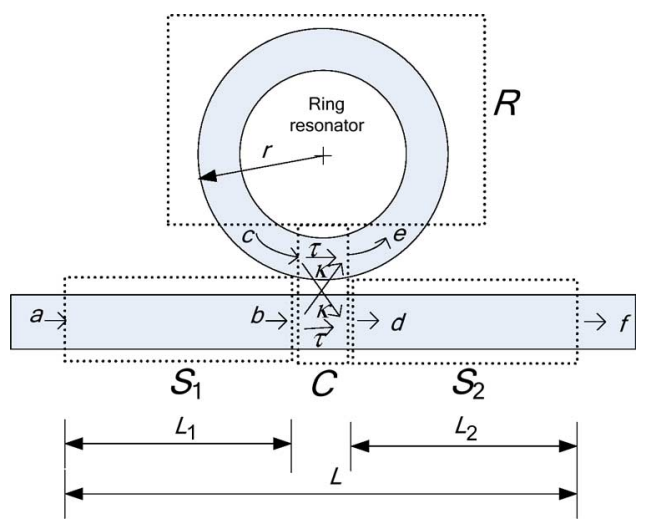

Fig. 1. TPRR circuit and the notations used in the modeling.

[9], [10]. Recently, Heebner and Boyd [9] briefly reported that a lossy two-port ring-resonator (TPRR) circuit, as depicted in Fig. 1, can also exhibit "fast" light with negative $v_{g}$ phenomenon when operated in an undercoupled condition. Negative group velocity in TPRR has also been studied for telecommunication applications [15], [16]. However, distortions and losses (see discussions in Section IV), which accompany such a phenomenon, together with the fact that no true signal "advancement" can be expected as $v_{g}$ is no longer the signal velocity [12] in such a phenomenon, could hinder its further exploitation. Until now, not much attention has been paid to taking advantage of light with negative $v_{g}$ phenomenon for applications such as sensing, nonlinear optics, and light generation/amplification.

In this paper, we model a single TPRR circuit, as shown in Fig. 1, and study the evolution of its group velocity from positive $v_{g}<c$, to negative $v_{g}$ with $\left|v_{g}\right|<c$, then to negative $v_{g}$ with $\left|v_{g}\right|>c$, then to positive $v_{g}>c$, and, finally, to positive $v_{g}<c$, again, as one varies the coupling constant from one extreme of full coupling to the other extreme of no coupling. Hence, we will show that in a TPRR circuit, light can either have a positive or negative $v_{g}$ and, in both situations, can either be "slow" $\left(\left|v_{g}\right|<c\right)$ or "fast" $\left(\left|v_{g}\right|>c\right)$. Note that our definitions of "fast" and "slow" are different from those widely used in the literature [1]. In the literature, the light need to have $v_{g} \ll c$ to be entitled as "slow" light, whereas for classification simplicity, in this paper, we simply denote $\left|v_{g}\right|<c$ as "slow" light. In our definition, light can either be "slow" or "fast" in the regime of negative $v_{g}$, whereas Boyd and Gauthier [1] always classify negative $v_{g}$ as "fast." The motivation for choosing different terms than the widely used ones will be given when 
discussing the simulation results. We analytically study the conditions for such operation regimes of $v_{g}$ in a lossy TPRR. We briefly discuss the nonrelevancy of negative $v_{g}$ and "fast" light in TPRR with gain. We also study the temporal behavior of pulses passing through the TPRR operated in those regimes, which shows pulse delaying, pulse "advancement," and pulse splitting. Moreover, we also study the sensitivity of phase shift to changes of the effective index of the ring $\left(S \equiv \partial \phi / \partial n_{\text {eff,res }}\right)$. We found that when the light is "slow," either with positive or negative $v_{g}$, there is enhancement of $S$, which suggests its potential for highly sensitive optical sensing applications.

\section{Modeling Method}

The TPRR circuit is divided into four parts, as shown in Fig. 1. In this paper, we assume that the coupler and straight waveguide sections are lossless, but the ring resonator can be lossy. We also assume that both the resonator and the straight waveguides are single-mode waveguides.

Assuming the time dependence of $\exp (i \omega t)$, the transfer function of the straight waveguide section for a wave traveling from left to right in Fig. 1 can be expressed as

$$
S_{1} \equiv \frac{b}{a}=\exp \left(-i \beta_{\text {straight }} L_{1}\right)
$$

and

$$
S_{2} \equiv \frac{f}{d}=\exp \left(-i \beta_{\text {straight }} L_{2}\right)
$$

where $a$ to $f$ are variables representing the modal fields at corresponding positions, as illustrated in the figure; $\beta_{\text {straight }}$, $L_{1}$, and $L_{2}$ are the propagation constant of the mode of the straight waveguide and the length of the first and the second sections of the straight waveguide, respectively.

The properties of the coupler section can be described using the following scattering matrix formulation:

$$
\left[\begin{array}{l}
d \\
e
\end{array}\right]=\mathbf{S}\left[\begin{array}{l}
b \\
c
\end{array}\right]
$$

where the scattering matrix

$$
\mathbf{S} \equiv\left[\begin{array}{cc}
\tau & \kappa \\
\kappa & \tau
\end{array}\right]
$$

with $\tau$ and $\kappa$ representing the through and cross-port amplitude coupling constant of the directional coupler, respectively. The transfer function of the ring is

$$
R \equiv \frac{c}{e}=\exp (-i \theta)
$$

with

$\theta=\left(\beta_{\text {res }}-i \alpha_{\text {res,lin }}\right) L_{\text {round-trip }}=\left(\beta_{\text {res }}-i \alpha_{\text {res,lin }}\right) 2 \pi r$

where $\beta_{\text {res }}, \alpha_{\text {res,lin }}, L_{\text {round-trip }}$, and $r$ represent the (linear) propagation constant, the attenuation constant (in linear scale), the effective round-trip propagation length, and the effective radius of the ring resonator, respectively. Using (3a) and (4), we obtain

$$
C \equiv \frac{d}{b}=\frac{\tau+\left(\kappa^{2}-\tau^{2}\right) \exp (-i \theta)}{1-\tau \exp (-i \theta)} .
$$

Hence, the transfer function of the TPRR circuit can be written as

$$
\begin{aligned}
T \equiv \frac{f}{a}=\frac{f}{d} \frac{d}{b} \frac{b}{a}=S_{2} C S_{1}= & \exp \left[-i \beta_{\text {straight }}\left(L_{1}+L_{2}\right)\right] \\
& \times \frac{\tau+\left(\kappa^{2}-\tau^{2}\right) \exp (-i \theta)}{1-\tau \exp (-i \theta)} .
\end{aligned}
$$

Using a coupled-mode theory analysis [17], it can be shown that $\kappa$ can be taken as a purely imaginary number and $\tau$ as a purely real number for a lossless directional coupler. By making use of the unitary property $\left(\mathbf{S S}^{H}=\mathbf{I}\right.$, where the superscript $H$ denotes transpose conjugation) of the scattering matrix as a consequence of power conservation of the lossless coupler, it can be shown that $\kappa^{2}-\tau^{2}=-|\kappa|^{2}-|\tau|^{2}=-1$. The transfer function of the structure can then be written as

$$
T=\exp \left(-i \beta_{\text {straight }} L\right) C
$$

where

$$
\begin{aligned}
L & =L_{1}+L_{2} \\
C & =\frac{\tau-\exp (-i \theta)}{1-\tau \exp (-i \theta)} .
\end{aligned}
$$

Note that the transfer function in (8) depends on the choice of $L$, which is rather arbitrary. The choice of $L$ influences calculated values such as the group index but does not change features such as the resonant wavelength. It is instructive to keep incorporating $L$ instead of doing normalization to the transfer function (such that it becomes independent on $L$ ), because in real devices, $L$ can be practically rather large for convenient handling.

Using a similar approach such as that of Benedickson et al. [18], by rewriting (8) as

$$
T=|T| \exp (-i \phi)
$$

we can calculate the group velocity and other phase-related parameters of the structure from the effective phase shift

$$
\phi=-\arctan \frac{\operatorname{Im}(T)}{\operatorname{Re}(T)} \pm 2 \pi p
$$

whereas the magnitude $|T|$ can be used to calculate the insertion loss of the structure. In (11), $p$ is an integer that plays no role in the following analysis.

\section{Classification of Operation Regimes OF GROUP VELOCITY}

In this section, we will use an analytical approach to get an insight into the behavior of $v_{g}$ in lossy and lossless TPRRs. We will also briefly comment on TPRRs with gain. We will classify the operation regimes of $v_{g}$ based on the analysis. 
For a ring resonator with loss, $\theta$ is a complex quantity, which allows us to write

$$
\exp (-i \theta)=\gamma \exp \left(-i \theta_{R}\right)
$$

with $\gamma \equiv \exp \left(\theta_{I}\right)$, where $\theta_{R}=2 \pi r \beta_{\text {res }}$ and $\theta_{I}=-2 \pi r \alpha_{\text {res,lin }}$ are the real and imaginary parts of $\theta$, respectively. Note that $0 \leq \gamma<1$ corresponds to a lossy structure, $\gamma=1$ corresponds to a lossless structure, and $\gamma>1$ corresponds to a structure with gain. Equation (9b) can then be rewritten as

$$
C=\frac{\tau-\gamma \exp \left(-i \theta_{R}\right)}{1-\tau \gamma \exp \left(-i \theta_{R}\right)}=|C| \exp \left(-i \phi_{c}\right)
$$

with

$$
\phi_{c}=-\arctan \left[\frac{\left(1-\tau^{2}\right) \gamma \sin \theta_{R}}{\tau\left(1+\gamma^{2}\right)-\left(1+\tau^{2}\right) \gamma \cos \theta_{R}}\right] .
$$

The total phase shift of the TPRR circuit is then

$$
\phi=\beta_{\text {straight }} L+\phi_{c}=\frac{\omega}{c} n_{\text {eff }, \text { straight }} L+\phi_{c}
$$

where $n_{\text {eff,straight }}$ is the effective index of the mode of the straight waveguide. The effective propagation constant of the TPRR circuit is then

$$
\beta_{\mathrm{eff}} \equiv \phi / L=\frac{\omega}{c} n_{\mathrm{eff}, \text { straight }}+\frac{\phi_{c}}{L} .
$$

By assuming that the coupling constant, ring attenuation constant, and effective indexes of the modes in the straight waveguide and the ring are not frequency dependent, the group velocity $v_{g} \equiv\left\{\partial \beta_{\text {eff }} / \partial \omega\right\}^{-1}$ can be written as

$$
v_{g}=\left\{\frac{n_{\text {eff }, \text { straight }}}{c}+\frac{1}{L} \frac{\partial \theta_{R}}{\partial \omega} \frac{\partial \phi_{c}}{\partial \theta_{R}}\right\}^{-1}
$$

where

$$
\frac{\partial \theta_{R}}{\partial \omega}=\frac{2 \pi r n_{\mathrm{eff}, \mathrm{res}}}{c}
$$

and

$$
\frac{\partial \phi_{c}}{\partial \theta_{R}}=\frac{\left(1-\tau^{2}\right) \gamma\left[\gamma\left(1+\tau^{2}\right)-\left(1+\gamma^{2}\right) \tau \cos \theta_{R}\right]}{\left(\tau^{2}-2 \gamma \tau \cos \theta_{R}+\gamma^{2}\right)\left(\tau^{2} \gamma^{2}-2 \tau \gamma \cos \theta_{R}+1\right)}
$$

where $n_{\text {eff,res }}$ is the (linear) effective index of the mode traveling in the ring resonator. Thus, the group index can be written as

$$
\begin{aligned}
n_{g} & \equiv \frac{c}{v_{g}}=n_{\mathrm{eff}, \text { straight }}+\frac{2 \pi r n_{\mathrm{eff}, \mathrm{res}}}{L} \\
& \times \frac{\left(1-\tau^{2}\right) \gamma\left[\gamma\left(1+\tau^{2}\right)-\left(1+\gamma^{2}\right) \tau \cos \theta_{R}\right]}{\left(\tau^{2}-2 \gamma \tau \cos \theta_{R}+\gamma^{2}\right)\left(\tau^{2} \gamma^{2}-2 \tau \gamma \cos \theta_{R}+1\right)} .
\end{aligned}
$$

For a lossless resonator, $\gamma=1$ leads (19) to

$$
\left.n_{g}\right|_{\gamma=1}=n_{\mathrm{eff}, \mathrm{straight}}+\frac{2 \pi r n_{\mathrm{eff}, \mathrm{res}}}{L} \frac{\left(1-\tau^{2}\right)}{\left(\tau^{2}-2 \tau \cos \theta_{R}+1\right)} .
$$

Note that since $-1 \leq \tau \leq 1$ and $-1 \leq \cos \theta_{R} \leq 1$, the second term of (20) is always positive. This fact implies that in a lossless TPRR circuit, $n_{g} \geq n_{\text {eff, straight }}>1$. Hence, in a lossless TPRR circuit, $v_{g}$ is always positive and smaller than $c$.

For structures with loss, since $-1 \leq \tau \leq 1,-1 \leq \cos \theta_{R} \leq 1$, and $\gamma>0$, the sign of the second term in (19) is determined by the sign of $A \equiv \gamma\left(1+\tau^{2}\right)-\left(1+\gamma^{2}\right) \tau \cos \theta_{R}$. The value of $A$ will be most negative when the structure is on resonance, i.e., when $\theta_{R}=2 \pi M$, with integer $M$, for $\tau>0$ or $\theta_{R}=2 \pi M-$ $\pi$ for $\tau<0$, which leads to

$$
\left.A\right|_{\theta_{R}=2 \pi M, \tau>0}=\gamma\left(1+\tau^{2}\right)-\left(1+\gamma^{2}\right) \tau=(\gamma-\tau)(1-\gamma \tau)
$$

and

$$
\left.A\right|_{\theta_{R}=2 \pi M-\pi, \tau<0}=\gamma\left(1+\tau^{2}\right)+\left(1+\gamma^{2}\right) \tau=(\gamma+\tau)(1+\gamma \tau) .
$$

At this resonance condition, the sign of $\left.A\right|_{\theta_{R}=2 \pi M, \tau>0}$ (or $\left.\left.A\right|_{\theta_{R}=2 \pi M-\pi, \tau<0}\right)$ will depend on the relationship between $\gamma$ and $\tau$ as follows.

1) The sign of $\left.A\right|_{\theta_{R}=2 \pi M, \tau>0}$ (or $\left.A\right|_{\theta_{R}=2 \pi M-\pi, \tau<0}$ ) will be negative if $|\tau|>\gamma$ (which is also known as the undercoupling condition). While $A$ is negative, the second part of (19) will be negative, leading to four possible conditions.

a) If the second part of (19) is much stronger than its first part, then $v_{g}$ is negative with $\left|n_{g}\right|>1$; hence, $\left|v_{g}\right|<c$. We refer this condition to a "slow" light with negative $v_{g}$.

b) If the second part of (19) is slightly stronger than its first part, such that $-1<n_{g}<0$, then negative $v_{g}$ with $\left|v_{g}\right|>c$ will happen. We refer this condition to a "fast" light with negative $v_{g}$.

c) If the second part of (19) is slightly weaker than its first part, such that $0<n_{g}<1$, then $v_{g}$ will be positive but superluminal $\left(v_{g}>c\right)$. We refer this condition to a "fast" light with positive $v_{g}$.

d) If the second part of (19) is weaker than its first part such that $n_{g}>1$, then the phenomenon of a positive $v_{g}$ that is smaller than $c$ will occur. We refer this condition to "slow" light with positive $v_{g}$. Note that for the weakest second part of (19), $n_{g}$ will be $n_{\text {eff, straight, }}$, which will limit the lowest $v_{g}$ in this regime.

Note that for lossless structures, since $\tau$ is always smaller than $\gamma, A$ is always positive; hence, there will be no possibility to have either superluminal or negative $v_{g}$ phenomena in such structure as has also been shown by (20). Also note that for a structure with gain, $\left.A\right|_{\theta_{R}=2 \pi M, \tau>0}$ (or $\left.A\right|_{\theta_{R}=2 \pi M-\pi, \tau<0}$ ) will be negative when $|\tau|>1 / \gamma$. Although this will lead to a similar effect as the undercoupling condition in lossy TPRR (hence one might think of similar phenomena in TPRR with gain), but in such $|\tau|>1 / \gamma$ condition, the field in the ring is not anymore stable as a result of net round-trip gain $|\tau| \gamma>1$. Hence, such "undercoupling" condition for structure with gain is not relevant for our model, 


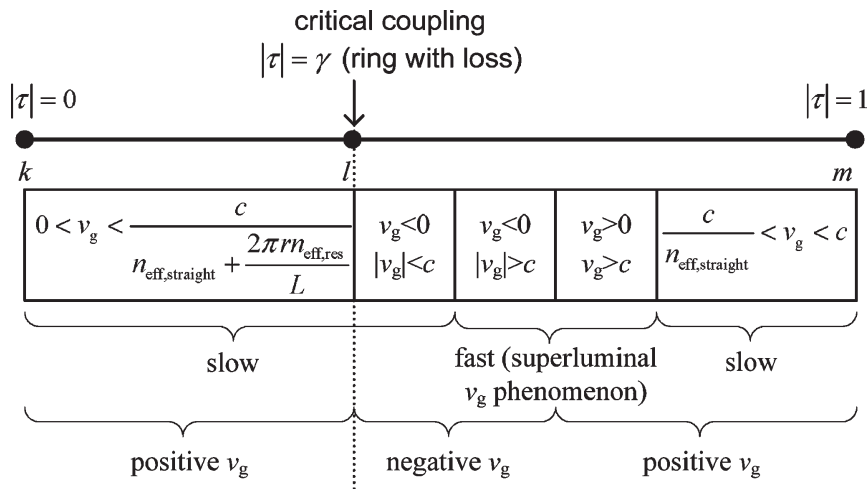

Fig. 2. Schematic map of $v_{g}$ at resonant frequency of the ring as one varies the $\tau$.

which relates the steady-state condition between the input and output of the TPRR.

2) For $|\tau|<\gamma$ in a lossy structure, or $|\tau|<1 / \gamma$ in a structure with gain, $A$ will always be positive, leading to $0<v_{g}<c /\left(n_{\mathrm{eff}, \text { straight }}+2 \pi r n_{\mathrm{eff}, \mathrm{res}} / L\right)$. Hence, at this condition, there will be no superluminal and negative $v_{g}$ phenomena. The light will always be "slow" with positive $v_{g}$. Note that for $\tau=0, n_{g}$ will be $n_{\text {eff, straight }}+$ $2 \pi r n_{\mathrm{eff}, \mathrm{res}} / L$, which will limit the highest $v_{g}$ in this regime. Also note that this overcoupling condition is still relevant for TPRR with gain.

Hence, it is clear that it is the loss that induces the superluminal and negative $v_{g}$ phenomena when operated under $|\tau|>\gamma$ condition. Such phenomena will most likely occur in the neighborhood of the resonant frequency of the resonator, where the value of $A$ is most negative and the second part of (19) is strongest (smallest denominator in $\partial \phi_{c} / \partial \theta_{R}$, hence most dispersive). A map of $v_{g}$ at resonant frequency when one varies $\tau$ is schematically shown in Fig. 2 as a summary of the aforementioned discussions. Note that point $l$ will move to the left for larger round-trip loss but to the right (widen the positive $v_{g}$ range at the left-hand side of the figure) as the round-trip loss becomes smaller until it coincides with point $m$ when the structure becomes lossless and removes the superluminal and negative $v_{g}$ regimes completely.

\section{RESUlts AND Discussions}

In this section, we will use the model that was discussed in Sections II and III to study the TPRR, including the transmission spectrum, $n_{g}$ spectrum, pulse temporal behavior, and phase sensitivity to ring effective index change $S$ in the operation regimes as discussed earlier. In this paper, we will take only positive $\tau$ since negative $\tau$ will give the same properties as has been discussed in the previous section.

Without loss of generality, for this paper, we consider TPRRs with $n_{\text {eff,straight }}, n_{\text {eff,res }}, r$, and $L$ of $2,2,300 \mu \mathrm{m}$, and $1 \mathrm{~cm}$, respectively. These values are chosen such that they are quite representative for realistic devices. Fig. 3 shows $n_{g}$ at the resonant frequency as a function of the coupling constant of lossy and lossless TPRRs, which have an attenuation constant $\alpha_{\text {res }}=20 \alpha_{\text {res, } \operatorname{lin}} \log (e)$ of $1 \mathrm{~dB} / \mathrm{cm}$ (corresponding to $\gamma=0.9785$ ) and $0 \mathrm{~dB} / \mathrm{cm}$, respectively. The figure clearly

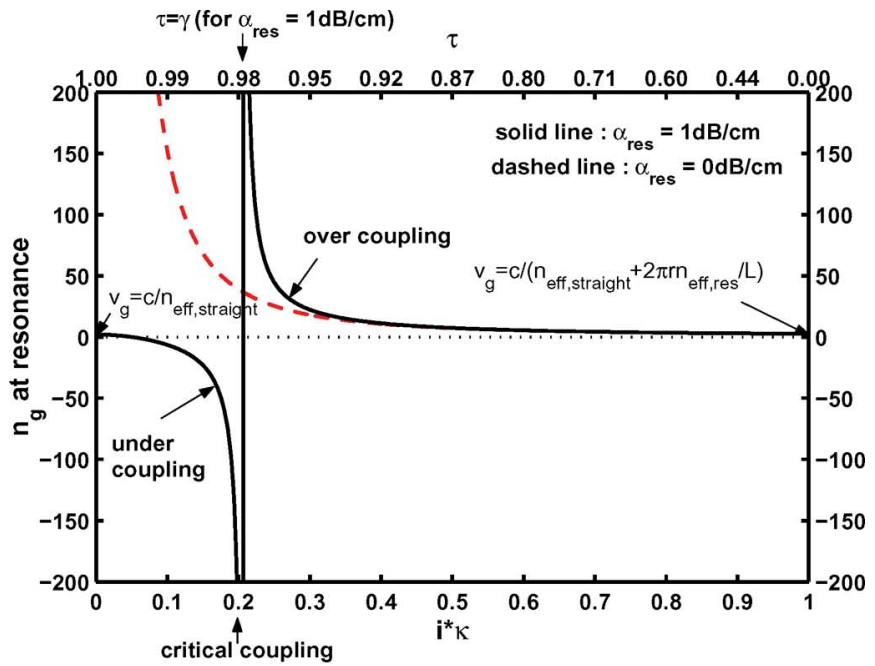

(a)

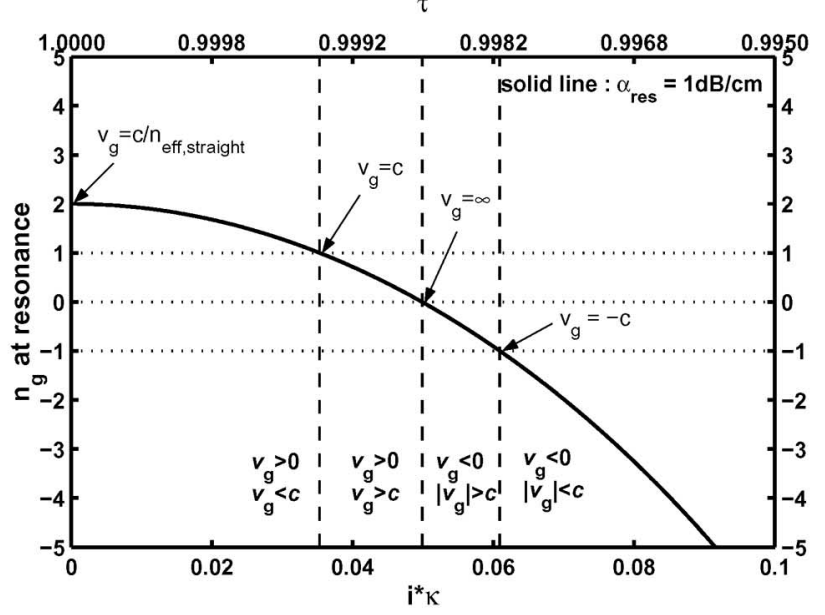

(b)

Fig. 3. (a) Plot of $n_{g}$ at resonant frequency as a function of the coupling constant for lossy and lossless TPRRs as specified in the text. (b) Zoomed plot of part of (a) showing the four possible regimes of $v_{g}$ in the undercoupling condition.

confirms the map given in Fig. 2. Note that in Fig. 3(b), there are regimes with positive $v_{g}$, although the ring resonator is already in the under-coupling condition, where it exhibits anomalous dispersion. This is because we have taken into account the effect of the straight waveguide for the reason explained in Section II, and hence, the overall properties of the device will depend on the balance between the positive and negative parts of (19), as detailed in Section III. We are aware that these properties (and hence the curves and position of the vertical dashed lines in Fig. 3) depend on the choice of $L$. Here, we have chosen a value of $L$, which is approximately the typical length of real device for typical laboratory experiments.

We then pick up samples of coupling constants corresponding to each operation regime of Fig. 3 and use (7) and Benedickson's approach to numerically calculate both the insertion loss and group index spectra of the structures for wavelengths in the neighborhood of $0.6328 \mu \mathrm{m}\left(\lambda_{\mathrm{HeNe}}\right)$.

First, we will show the superluminal $v_{g}$ phenomenon for the lossy TPRR, both with positive and negative $v_{g}$ at the 


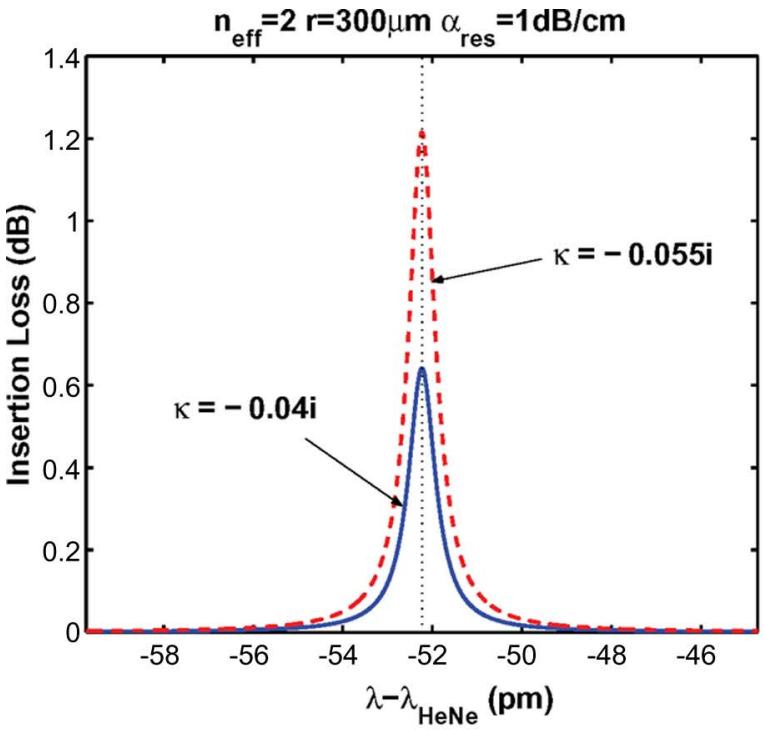

(a)

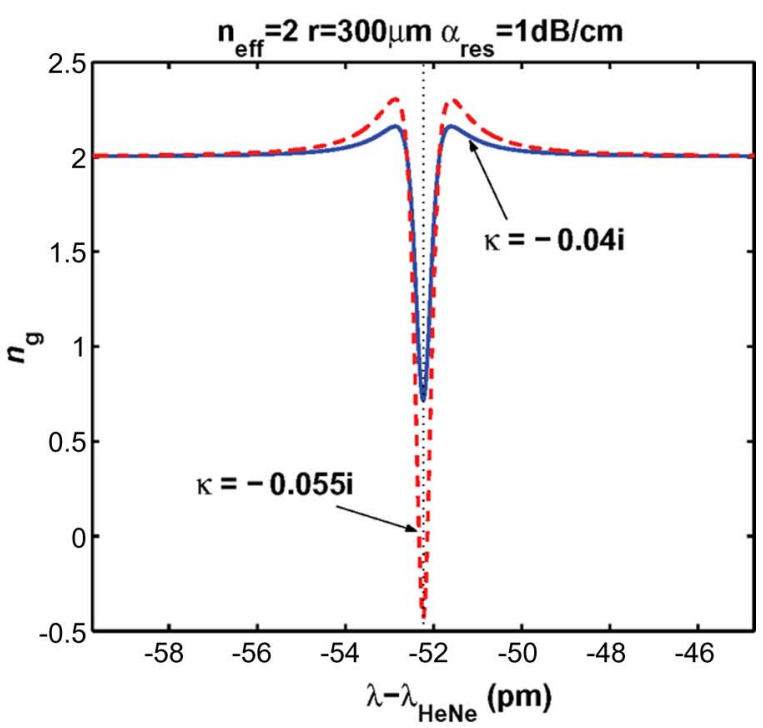

(b)

Fig. 4. (a) Insertion loss and (b) group index of the lossy TPRR circuit with $\kappa=-0.04 i$ and $-0.055 i$. The other parameters are as specified in the text. At the resonant frequency of the resonator, the circuit operates in the "fast" light with positive $v_{g}$ (i.e., $v_{g}>c$ ) and the "fast" light with negative $v_{g}$ (i.e., $v_{g}<0$ and $\left|v_{g}\right|>c$ ) regimes, respectively.

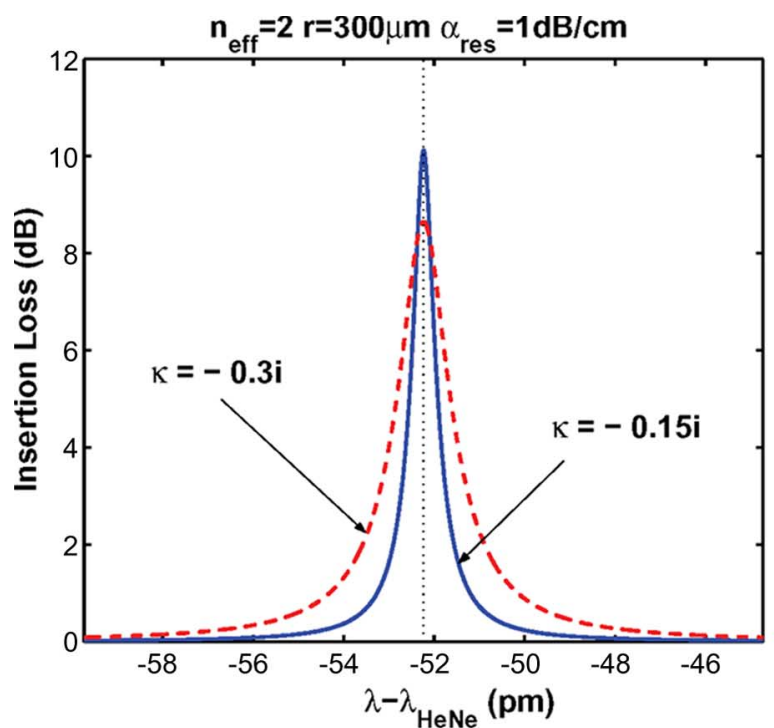

(a)

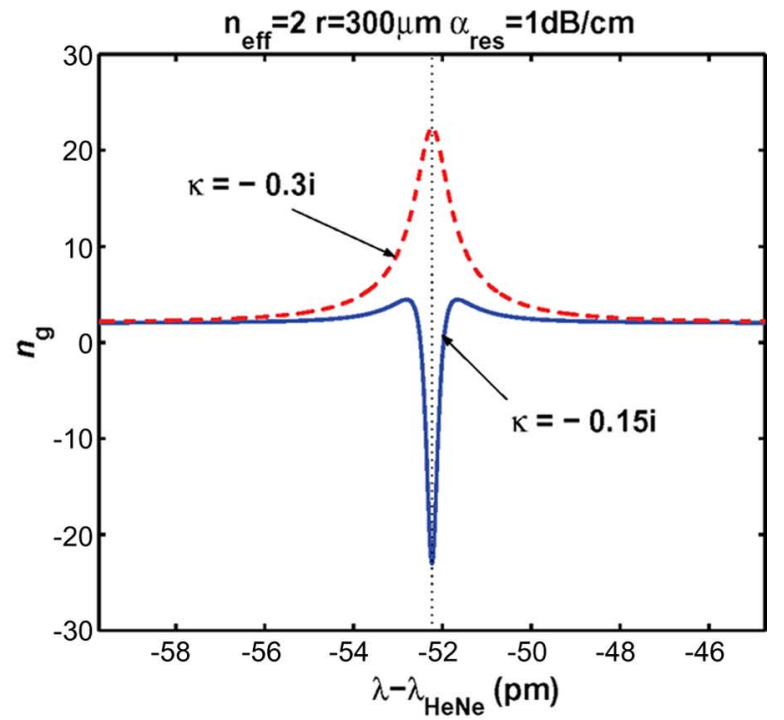

(b)

Fig. 5. (a) Insertion loss and (b) group index of a lossy TPRR circuit with $\kappa=-0.15 i$ and $-0.3 i$. The other parameters are as specified in the text. At the resonant frequency of the resonator, the circuit operates in the "slow" light with negative $v_{g}$ (i.e., $v_{g}<0$ and $\left|v_{g}\right|<c$ ) and the "slow" light with positive $v_{g}$ (i.e., $0<v_{g}<c$ ) regimes, respectively.

resonant frequency of the resonator. Fig. 4 shows the results for $\kappa=-i 0.04$ and $\kappa=-i 0.055$, which are associated to the "fast" light with positive $v_{g}$ and the "fast" light with negative $v_{g}$ regimes, respectively. Note that since the light spends only a very short time in the resonator (as it is "fast"), the light-matter interaction is low in such superluminal $v_{g}$ phenomena; hence, the total insertion loss is relatively small.

Results corresponding to "slow" propagation of light $\left(\left|v_{g}\right|<\right.$ $c$ ), both with negative and positive $v_{g}$, are shown in Fig. 5 . The slower the light, the more time the light spends in the resonator, the more the light-matter interaction is, hence the larger the insertion loss is exhibited by the structure. This fact suggests that the slowest achievable $v_{g}$ in real application with passive TPRR will be limited by the maximum tolerable insertion loss in the structure, as "too slow" light will result in too small output intensity, which might then be dominated by noise. Such light-matter interaction behavior applies for both "slow" light with a positive $v_{g}$ and a negative $v_{g}$.

To study the temporal behavior of pulses and to further illustrate the phenomena, we take a pulse with Gaussian envelope as follows:

$$
a(t)=\exp \left(-t^{2} / t_{d}^{2}\right) \exp \left(i \omega_{c} t\right)
$$




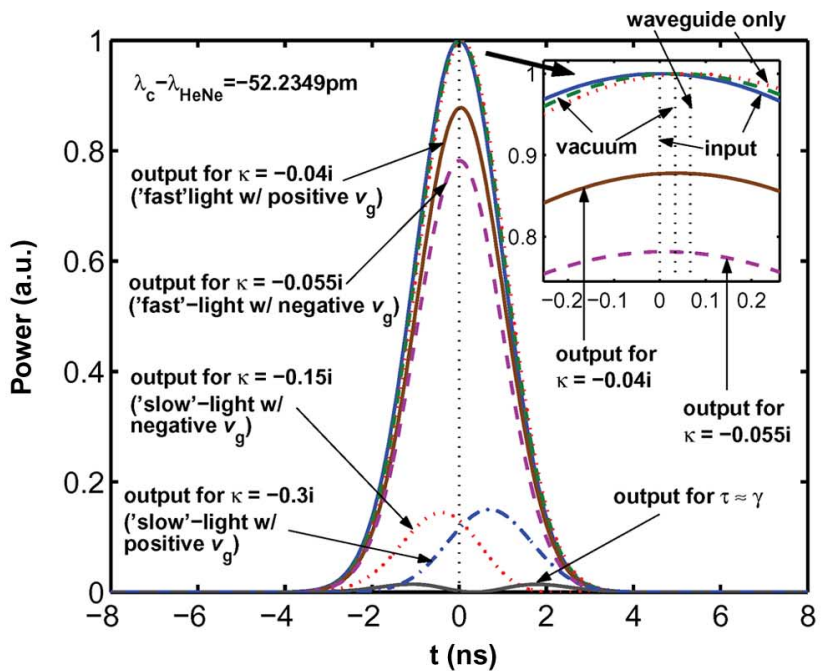

(a)

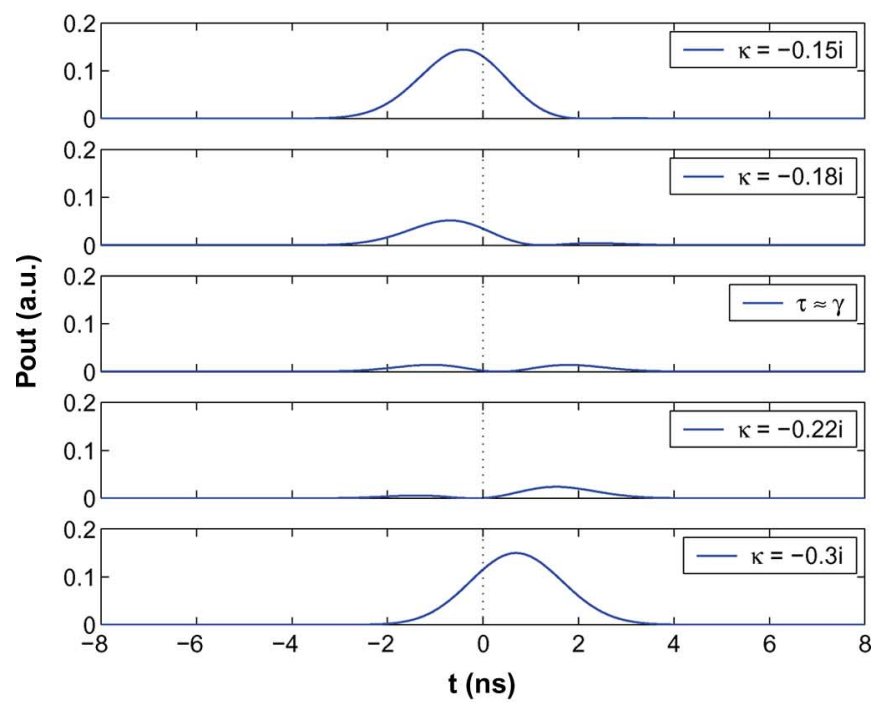

(b)

Fig. 6. (a) Power of pulses at the output of a lossy TPRR circuit if a Gaussian pulse is injected into the input of the TPRR circuit operated in various regimes. For reference, the input pulse, the output pulse if it would travel through vacuum of length $L$, and the output pulse if it would travel through a straight waveguide only (without ring resonator) of length $L$ are also plotted together with vertical dotted lines to indicate their peak positions. (b) Smooth transition from a negative to a positive delay.

with $t_{d}=2 \mathrm{~ns}$ and $\omega_{c}=2 \pi c / \lambda_{c}$, where $\lambda_{c}$ is the resonant wavelength nearest to $\lambda_{\mathrm{HeNe}}$, and inject the pulse into the input of TPRRs working in various operation regimes, as given in Figs. 4 and 5. By using Fourier transform, transfer function (7), and inverse Fourier transform subsequently, the shape of output pulse can be observed. Fig. 6(a) shows the power of output pulses for various operation regimes of the TPRRs. For reference, we also plot the input pulse, the output pulse if it would travel through vacuum with length $L$, and the output pulse if it would travel through the straight waveguide only (without ring resonator) with length $L$. Here, we have assumed that the straight waveguide is not dispersive. For the "fast" light regimes, the pulses need only small transit times, and hence experience only small phase shifts and small insertion losses. Thus, apart from scientific curiosity, it is not clear

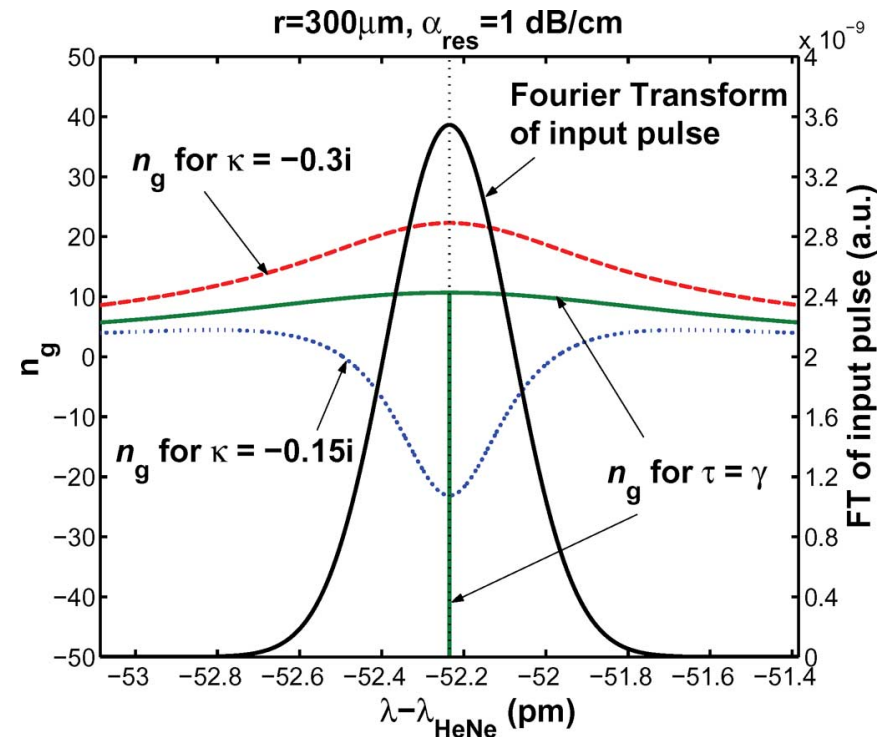

Fig. 7. If the TPRR circuit is operated in the neighborhood of $\tau=\gamma$, the spectrum of $n_{g}$ becomes very sharp (note the sharp, "negative delta-function"like dip in the figure) such that the spectrum of the input pulse covers the highly dispersive spectrum of the ring well. This situation makes the pulse to be highly distorted, and it splits into two output pulses-one with positive and the other with negative delay-as shown in Fig. 6. The $n_{g}$ curves for $\kappa=-0.15 i$ ("slow" light with negative $v_{g}$ at resonance) and $-0.3 \mathrm{i}$ ("slow" light with positive $v_{g}$ at resonance) are also shown for reference.

yet what applications can take advantage of such "fast" light phenomenon since the light-matter interaction is low.

Fig. 6(a) shows that for "slow" light regimes, the pulses experience considerable delay and insertion loss. For "slow" light with positive $v_{g}$, the pulse experiences positive delay, whereas for "slow" light with negative $v_{g}$, the pulse experiences negative delay, where the peak of the output pulse appears earlier than the peak of the input pulse. Since the leading edge of the input pulse already entered the device before the relatively weak output pulse with negative $v_{g}$ coming out of the device, the energy of such output pulse indeed comes from the energy of the input pulse, as has been discussed in the literature [1]. Moreover, the leading edge of the output pulse if it would travel through vacuum of the same length also already left its output before the output pulse with negative $v_{g}$ left the device. Hence, there is no violation of the causality. More detailed discussions on the causality of superluminal and negative $v_{g}$ phenomena can be found in the literature [5], [19]. Note that both the "slow" light with positive $v_{g}$ and the "slow" light with negative $v_{g}$ have similar properties, i.e., strong light-matter interaction, high insertion loss, and large delay, where the delay is positive for "slow" light with positive $v_{g}$ but negative for "slow" light with negative $v_{g}$. The larger the delay, the higher the insertion loss is. Such similarities are the reasons that motivate us to term the phenomenon corresponding to $v_{g}<0$ and $\left|v_{g}\right|<c$ as "slow" light instead of "fast" light. We believe that, as one can take advantages of the intense light-matter interaction in the "slow" light with positive $v_{g}$ for certain applications, similar advantages can also be expected to some extent for "slow" light with negative $v_{g}$.

It is also interesting to observe that when the TPRR circuit is operated in the neighborhood of $\tau=\gamma$ (critical coupling 


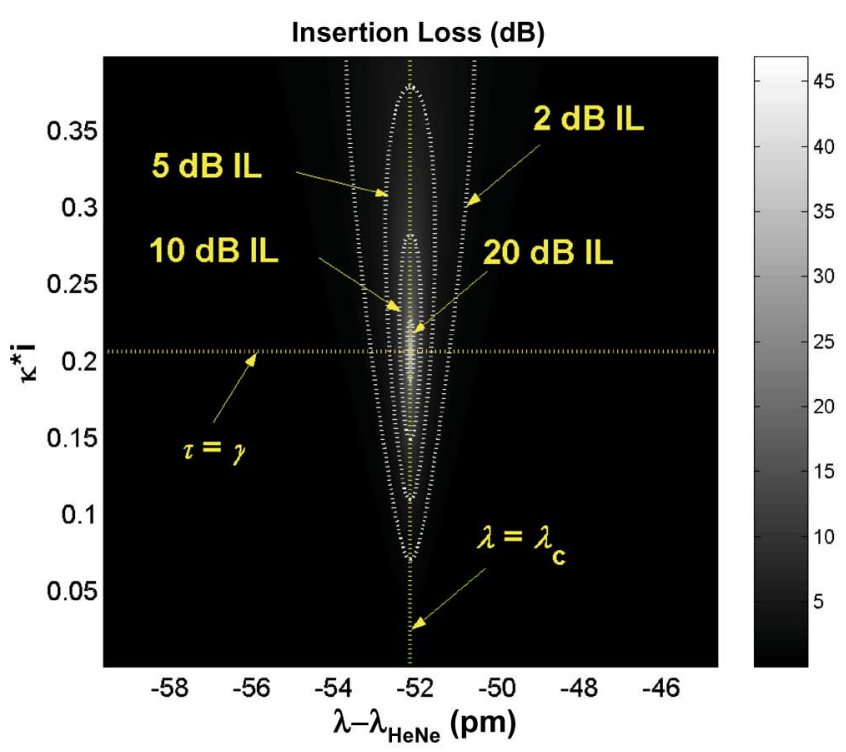

(a)

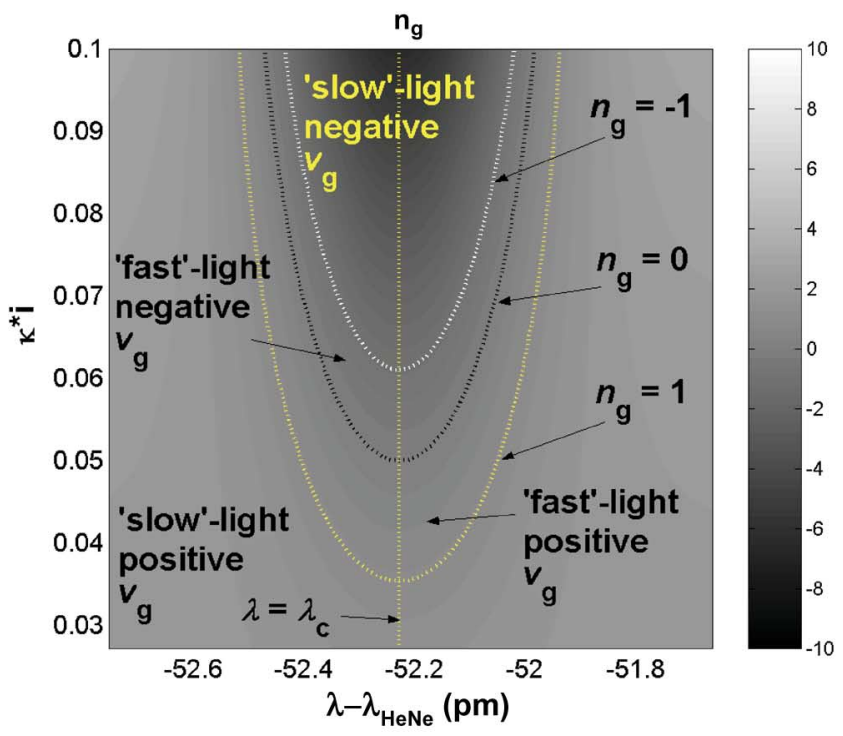

(c)

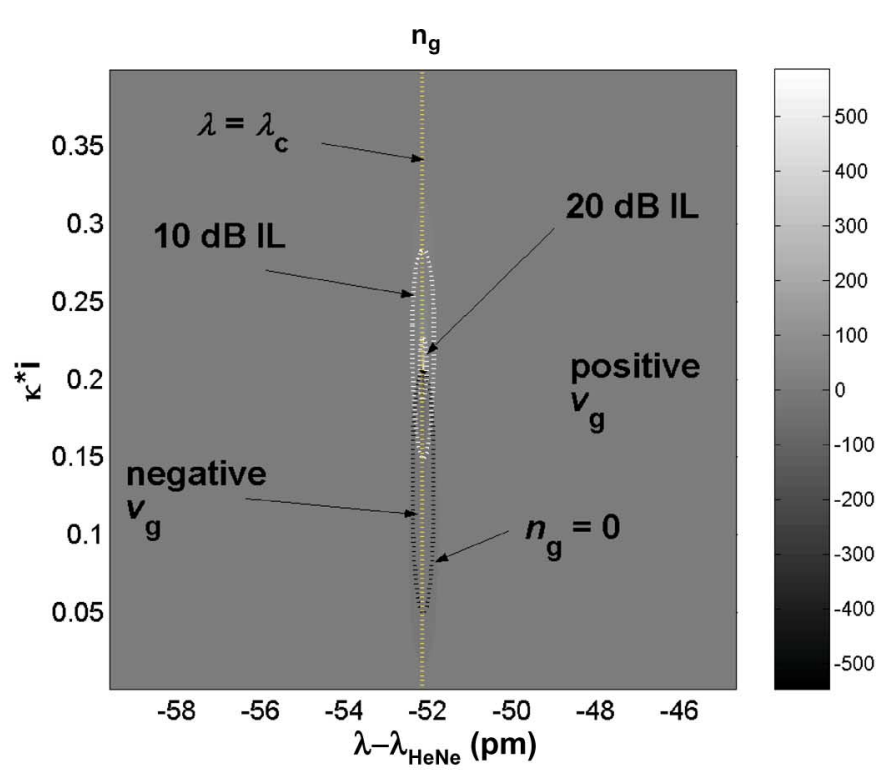

(b)

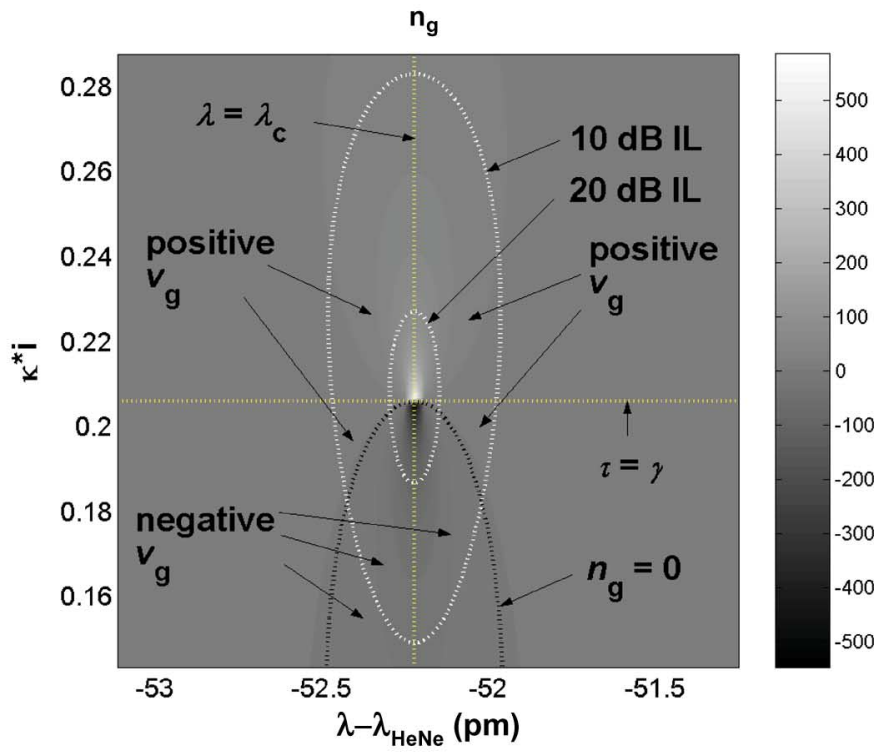

(d)

Fig. 8. Map of (a) insertion loss and (b) group index as one varies both the wavelength and the coupling constant of a TPRR with an attenuation coefficient of $1 \mathrm{~dB} / \mathrm{cm}$ in the ring. The other parameters are as specified in the text. (c) and (d) Zoomed pictures of (b) showing the regimes with "fast" light and "slow" light, positive and negative $v_{g}$. The contour lines of insertion loss of 10 and $20 \mathrm{~dB}$ of (a) are superimposed into (b) and (d) to show that the large delay (either with positive of negative $v_{g}$ ) comes at the expense of a high insertion loss as a consequence of a high light-matter interaction.

condition), the single input pulse splits into two output pulses, one with positive and one with negative delay. This phenomenon is attributed to the fact that the input pulse is not anymore monochromatic due to the modulation, and its spectrum is wide enough to cover the highly dispersive part of the TPRR response curve. Consequently, the phase relation between the Fourier components in that frequency range is also heavily changed. Such a situation makes the pulse to be highly distorted and apparently split into two output pulses. Although the $n_{g}$ curve [see Fig. 3(a)] changes abruptly when one varies the coupling constant across the critical coupling point, such pulse splitting happens in a rather smooth manner, resulting in a smooth transition from a negative to a positive delayed pulse, or vice versa [see Fig. 6(b)]. It is worth to note that the slower the light, the higher the pulse distortion is, which results in losses of the pulse analog information. Fig. 7 shows that the $n_{g}$ spectrum of TPRR with negative $v_{g}$ at resonance (e.g., for $\kappa=-0.15 i$ ) contains both the positive and negative $n_{g}$ parts, whereas the one of TPRR with positive $v_{g}$ at resonance (e.g., for $\kappa=-0.3 i$ ) only contains the positive $n_{g}$ part. Such difference in complexity in $n_{g}$ spectrum suggests that the distortion for "slow" light with negative $v_{g}$ at resonance is higher than the distortion for "slow" light with positive $v_{g}$ at resonance. This pulse distortion will limit the usefulness of the large negative $v_{g}$, which is obtained, for example, by cascading several TPRR circuits. Recently, the pulse temporal behavior, as shown in 
Fig. 6 and as previously discussed, has been experimentally verified and is reported elsewhere [20], [21].

In Fig. 8, we show the map of the insertion loss and group index as one varies the wavelength and coupling constant of the lossy TPRR. The pictures clearly show that the interesting operation regimes of the TPRR occur around the resonant wavelength, where the structure is most dispersive. The structure is also the slowest (has lowest $v_{g}$ ) but has the most insertion loss at the critical coupling $(\tau=\gamma)$ condition at the resonant wavelength as the light-matter interaction is the strongest.

The intensive light-matter interaction for "slow" light either with positive or negative $v_{g}$ suggests its potential for sensing applications. At present, since one of the most sensitive integrated optical sensing readout schemes is a phase shift readout scheme [22], we will briefly comment about the possibility of using the TPRR both with "slow" positive and negative $v_{g}$ for sensing applications with this scheme. For simplicity, we will consider refractometric sensing. One possible approach is by using the analyte as the cladding of the ring waveguide and hence being interrogated by the evanescent field of the light circling in the ring and influencing its effective index $\left(n_{\text {eff,res }}\right)$. The phase change of light passing through the TPRR will then be read out (e.g., using a serrodyne modulation and interferometric approach [22]) as a measure of the refractive index change of the analyte. The sensitivity of phase shifts to the changes of the effective index of the ring resonator $S \equiv \partial \phi / \partial n_{\text {eff,res }}$ and the sensitivity of the ring effective index to the changes of the analyte refractive index $\partial n_{\text {eff,res }} / \partial n_{\text {mea }}$ will then determine the sensitivity of the sensor. Fig. 9(a) shows the $S$ for the TPRR for the four operation regimes, as discussed earlier. The figure clearly shows that when the light is "slow," there is enhancement of $S$. By writing $|S| \equiv k_{0} L_{\text {eff }}$, where $k_{0}$ and $L_{\text {eff }}$ are the free space wavenumber and the effective sensor interaction length, respectively, we can calculate $L_{\text {eff. Fig. 9(b) }}$ shows such effective sensor interaction length. For the "slow" light setting with positive $v_{g}(\kappa=-0.3 i)$ and negative $v_{g}$ $(\kappa=-0.15 i)$, the $L_{\text {eff }}$ are around 11.1 and $11.6 \mathrm{~cm}$, respectively, by using a TPRR with a single ring resonator with a radius of only $300 \mu \mathrm{m}$ operated at its resonant wavelength. By taking typical values of $\partial n_{\text {eff,res }} / \partial n_{\text {mea }}$ and minimum phase detection limit $\delta \phi$ of 0.2 and $5 \mathrm{E}-5 \times 2 \pi \mathrm{rad}$. [23], respectively, these two "slow" light settings already offer refractive index detection limit $\delta n_{\text {mea }}$, i.e.,

$$
\delta n_{\text {mea }}=\frac{\delta \phi}{S \partial n_{\text {eff,res }} / \partial n_{\text {mea }}}
$$

of around $1.5 E-9$ refractive index units, which is quite an impressive number. The insertion losses [as given in Fig. 5(a)] are around 8.6 and $10 \mathrm{~dB}$, respectively. We should note that these settings have not been optimized yet, because in typical sensing applications, we can opt for larger insertion loss. We should also note that for such phase readout scheme, there is a tradeoff between the slowness of the light and the insertion loss. These results indicate that "slow" light with negative $v_{g}$ (as well as the one with positive $v_{g}$ ) can be exploited for highly sensitive optical sensors.

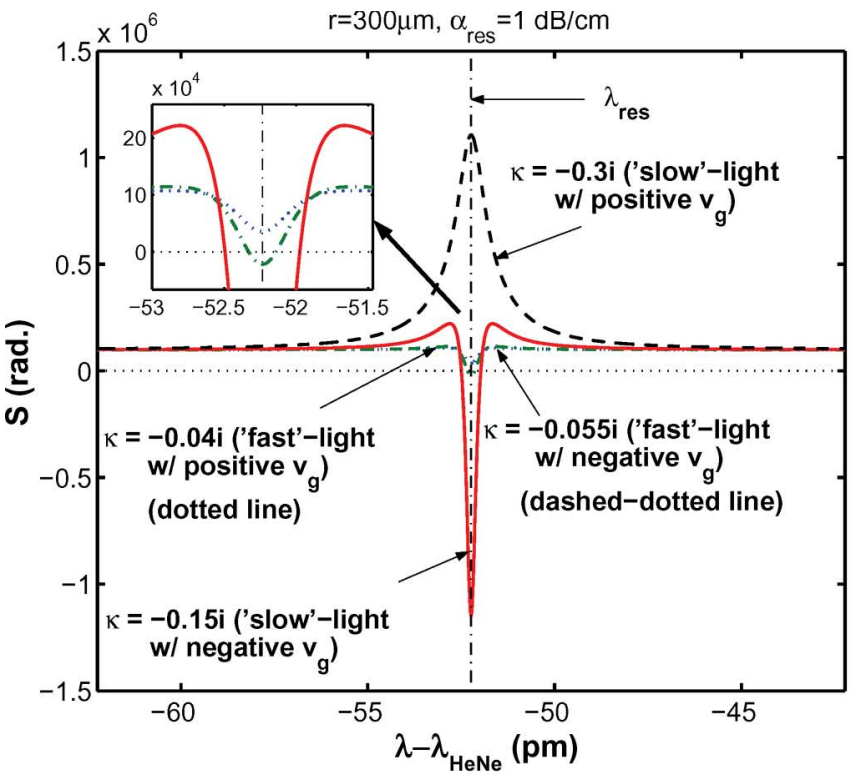

(a)

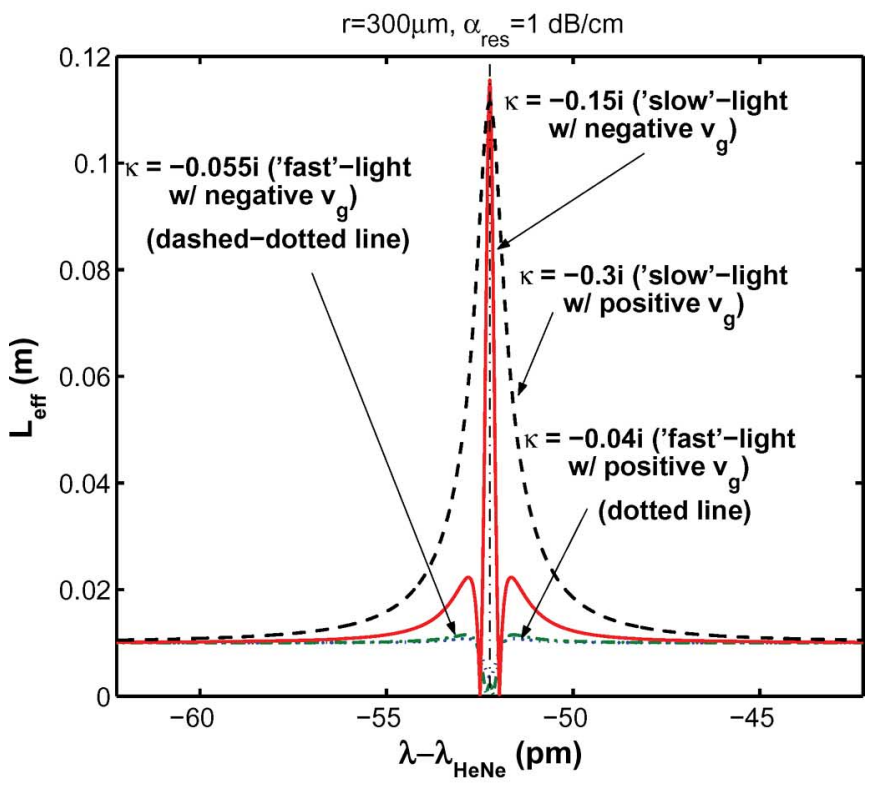

(b)

Fig. 9. (a) Sensitivity of the phase shift to the changes in the effective index of the resonator. (b) Corresponding effective sensor interaction length.

\section{CONCLUSion}

The behavior of $v_{g}$ of light in TPRR circuits with loss is modeled and studied. Using an analytical approach, we point out and classify the light properties into "slow" light with positive $v_{g}$, "slow" light with negative $v_{g}$, "fast" light with negative $v_{g}$, and "fast" light with positive $v_{g}$. The temporal behavior of pulses traveling through the TPRR operating in various operation regimes is also studied, showing pulse delaying, pulse "advancement," and pulse splitting. We show that when the light is "slow," either with positive or negative $v_{g}$, there is an enhanced light-matter interaction, which is indicated by a large loss. The possible application of such enhanced light-matter interaction for sensing is also briefly discussed. 


\section{ACKNOWLEDGMENT}

The authors would like to thank R. M. de Ridder, M. Hammer, and R. Stoffer for discussions.

\section{REFERENCES}

[1] R. W. Boyd and D. J. Gauthier, "Slow' and 'fast' light," in Prog. in Optics, vol. 43, E. Wolf, Ed. Amsterdam, The Netherlands: Elsevier, 2002, pp. 497-530.

[2] J. Scheuer, G. T. Paloczi, J. K. S. Poon, and A. Yariv, "Coupled resonator optical waveguides: Toward the slowing \& storage of light," Opt. Photon. News, vol. 16, no. 2, pp. 36-40, 2005.

[3] F. R. Faxvog, C. N. Y. Chow, T. Bieber, and J. A. Carruthers, "Measured pulse velocity greater than $c$ in a neon absorption cell," Appl. Phys. Lett., vol. 17, no. 5, pp. 192-193, Sep. 1970.

[4] L. J. Wang, A. Kuzmich, and A. Dogariu, "Gain-assisted superluminal light propagation," Nature, vol. 406, no. 6793, pp. 277-279, Jul. 2000.

[5] M. D. Stenner, D. J. Gauthier, and M. A. Neifeld, "The speed of information in a "fast-light" optical medium," Nature, vol. 425, no. 6959, pp. 695698, Oct. 2003.

[6] K. Y. Song, M. G. Herráez, and L. Thévenaz, "Observation of pulse delaying and advancement in optical fibers using stimulated Brillouin scattering," Opt. Express, vol. 13, no. 1, pp. 82-88, Jan. 2005.

[7] L. V. Hau, S. E. Harris, Z. Dutton, and C. H. Behroozi, "Light speed reduction to 17 metres per second in an ultracold atomic gas," Nature, vol. 397, no. 6720, pp. 594-598, Feb. 1999.

[8] A. M. Steinberg, P. G. Kwiat, and R. Y. Chiao, "Measurement of the single-photon tunneling time," Phys. Rev. Lett., vol. 71, no. 5, pp. 708711, Aug. 1993.

[9] J. E. Heebner and R. W. Boyd, "Slow and fast light in resonator-coupled waveguides," J. Mod. Opt., vol. 49, no. 14/15, pp. 2629-2636, 2002.

[10] A. Melloni, F. Morichetti, and M. Martinelli, "Linear and nonlinear pulse propagation in coupled resonator slow-wave optical structures," Opt. Quantum Electron., vol. 35, no. 4/5, pp. 365-379, Mar. 2003.

[11] H. Altug and J. Vuckovic, "Experimental demonstration of the slow group velocity of light in two-dimensional coupled photonic crystal microcavity arrays," Appl. Phys. Lett., vol. 86, no. 11, pp. $111102.1-111102.3$, Mar. 2005.

[12] L. Brillouin, Wave Propagation and Group Velocity. New York: Academic, 1960.

[13] C. G. B. Garrett and D. E. McCumber, "Propagation of a Gaussian light pulse through an anomalous dispersion medium," Phys. Rev. A, Gen. Phys., vol. 1, no. 2, pp. 305-313, Feb. 1970.

[14] J. F. Woodley and M. Mojahedi, "Negative group velocity and group delay in left-handed media," Phys. Rev. E, Stat. Phys. Plasmas Fluids Relat. Interdiscip. Top., vol. 70, no. 4, pp. 046 603.1-046 603.6, Oct. 2004.

[15] H. Takahashi, P. Carlsson, K. Nishimura, and M. Usami, "Analysis of negative group delay response of all-pass ring resonator with Mach-Zehnder interferometer," IEEE Photon. Technol. Lett., vol. 16, no. 9, pp. 20632065, Sep. 2004.

[16] L. Zhuang, "Time-delay properties of optical ring resonators," M.S. thesis, Univ. Twente, Enschede, The Netherlands, 2005.

[17] C. K. Madsen and J. H. Zhao, Optical Filter Design and Analysis: A Signal Processing Approach. New York: Wiley, 1999.

[18] J. M. Benedickson, J. P. Dowling, and M. Scalora, "Analytic expressions for the electromagnetic mode density in finite, one-dimensional, photonic band-gap structure," Phys. Rev. E, Stat. Phys. Plasmas Fluids Relat. Interdiscip. Top., vol. 53, no. 4, pp. 4107-4121, Apr. 1996.
[19] M. Mojahedi, K. J. Malloy, G. V. Eleftheriades, J. Woodley, and R. Y. Chiao, "Abnormal wave propagation in passive media," IEEE J. Sel. Topics Quantum Electron., vol. 19, no. 1, pp. 30-39, Jan./Feb. 2003.

[20] H. P. Uranus, L. Zhuang, C. G. H. Roeloffzen, and H. J. W. M. Hoekstra, "Direct experimental observation of pulse temporal behavior in integrated-optical ring-resonator with negative group velocity," presented at the Eur. Conf. Integrated Optics (ECIO), Copenhagen, Denmark, Apr. 25-27, 2007, Post Deadline Paper FPD1.

[21] H. P. Uranus, L. Zhuang, C. G. H. Roeloffzen, and H. J. W. M. Hoekstra, "Pulse advancement and delay in an integrated optical two-port ring-resonator circuit: Direct experimental observations," Opt. Lett., to be published.

[22] P. V. Lambeck, "Integrated optical sensors for the chemical domain," Meas. Sci. Technol., vol. 17, no. 8, pp. R93-R116, Aug. 2006.

[23] R. G. Heideman and P. V. Lambeck, "Remote opto-chemical sensing with extreme sensitivity: Design, fabrication and performance of pigtailed integrated optical phase-modulated Mach-Zehnder interferometer system," Sens. Actuators B, Chem., vol. 61, no. 1-3, pp. 100-127, Dec. 1999.

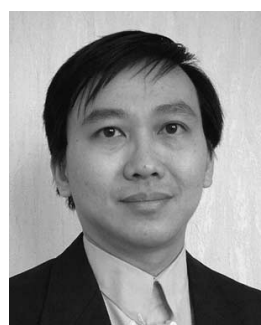

H. P. Uranus received the Engineer (Ir.) degree in electrical engineering from Trisakti University, Jakarta, Indonesia, in 1987, the Master's degree in optoelectronics from the University of Indonesia, Jakarta, in 1990, and the Ph.D. degree in modeling of integrated optics from the University of Twente, Enschede, The Netherlands, in 2005.

From 1987 to 2001, he was an Engineer with CV Trans Komunikasi Data, Jakarta. From 1990 to 2001 and 1997 to 2001, he was a part-time Junior Lecturer with the University of Indonesia and the University of Pelita Harapan, Jakarta. Since 2005, he has been a Postdoctoral Researcher with the University of Twente, where he works on photonic sensors. His interests are in modeling, realization, and characterization of guided-wave and leaky wave photonic devices.

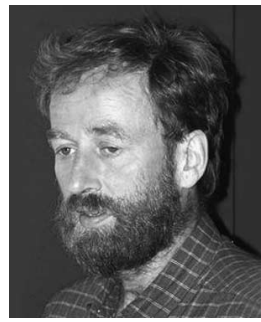

H. J. W. M. Hoekstra, received the $\mathrm{Ph}$. D. degree from the University of Groningen, Groningen, Germany, in 1984, where he worked on solid-state physics.

Currently, he is with the Integrated Optical MicroSystems Group, MESA+ Institute for Nanotechnology, University of Twente, Enschede, The Netherlands, focusing on integrated optics sensors for multisensing applications in biochemistry and trace-gas sensing. 